\title{
Abundant novel solitary wave Solutions of two-mode Sawada-Kotera model and its Applications
}

\author{
Ammar Oad ${ }^{1}$, Muhammad Arshad ${ }^{2}$, Muhammad Shoaib ${ }^{3}$, Dianchen Lu², and Li Xiaohong ${ }^{1}$ \\ ${ }^{1}$ Shaoyang University \\ ${ }^{2}$ Jiangsu University \\ ${ }^{3}$ Hunan University
}

June 20, 2021

\begin{abstract}
The Sawada-Kotera equations illustrating the non-linear wave phenomena in shallow water, ion-acoustic waves in plasmas, fluid dynamics etc. In this article, the two-mode Sawada-Kotera equation (tmSKE) occurring in fluid dynamics is addresses. The improved F-expansion and generalized $\exp \$(-\backslash$ phi $(\backslash$ zeta $)) \$$-expansion methods are utilized in this model and abundant of solitary wave solutions of different kinds such as bright and dark solitons, multi peak soliton, breather type waves, periodic solutions and other wave results are obtained. These achieved abundant novel solitary and other wave results have significant applications in fluid dynamics, applied sciences and engineering. By granting appropriate values to parameters, the structures of few results are presented in which many structures are novel. The graphical moments of few solutions helps the engineers and scientist for understanding the physical phenomena of this model. To explain the novelty between the present results and the previously attained results, a comparative study has been carried out. Furthermore, the executed techniques can be employed for further studies to explain the realistic phenomena arising in fluid dynamics correlated with any physical and engineering problems.
\end{abstract}

\section{Hosted file}

Research Paper.pdf available at https://authorea.com/users/420656/articles/526960-abundantnovel-solitary-wave-solutions-of-two-mode-sawada-kotera-model-and-its-applications 